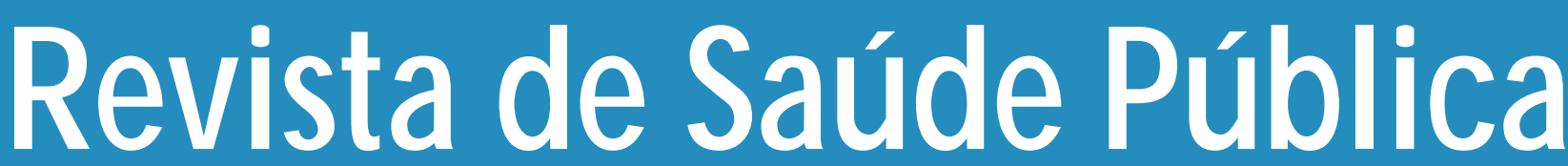
Journal of Public Health

\title{
Envelhecimento funcional e condições de traba- Iho em servidores forenses
}

Aging and work conditions in forensic workers

\section{Silvia Meirelles Bellusci e Frida Marina Fischer}

Departamento de Saúde Ambiental da Faculdade de Saúde Pública da Universidade de São Paulo. São Paulo, SP - Brasil 


\title{
Envelhecimento funcional e condições de trabalho em servidores forenses* Aging and work conditions in forensic workers
}

\author{
Silvia Meirelles Bellusci** e Frida Marina Fischer \\ Departamento de Saúde Ambiental da Faculdade de Saúde Pública da Universidade de São Paulo. São \\ Paulo, SP - Brasil
}

\section{Descritores}

Envelhecimento. Avaliação da capacidade de trabalho.

Ergonomia (saúde ambiental). Setor público.

\section{Keywords}

Aging. Work capacity evaluation.

Ergonomics (environmental

health). Public sector.

\section{Resumo}

\section{Objetivo}

Avaliar o envelhecimento funcional (capacidade para o trabalho) associado às condições de trabalho.

\section{Métodos}

Responderam ao questionário "Índice de Capacidade para o Trabalho" (ICT), 807 servidores de uma instituição judiciária federal. As condições de trabalho foram analisadas através do método de análise ergonômica do trabalho.

\section{Resultados}

A maioria das funções estudadas tem predomínio de demandas cognitivas no trabalho. Os diagnósticos mais referidos foram: doenças músculo-esqueléticas (e lesões), neurológicas (incluindo distúrbio emocional), respiratórias, digestivas, dermatológicas e cardiovasculares. Os modelos de análise de regressão logística mostraram que as mulheres, aqueles com maior tempo de trabalho na instituição e os com cargo de auxiliar operacional de serviços diversos têm maiores chances de apresentar ICT baixo ou moderado.

Conclusões

Os resultados ressaltam a necessidade de melhorar as condições de trabalho. Sugerese a implementação do Serviço Especializado em Engenharia de Segurança e Medicina do Trabalho, como exige a Lei 6.514 de 1977.

\footnotetext{
Abstract

Objective

The objective of this study was to evaluate aging associated to work conditions. Method

Eight hundred and seven forensic workers answered the questionnaire "Work Ability Index - WAI". The ergonomic conditions were analysed using a job analysis method - AET (Rohmert \& Landau).

Results

The largest number of employees were mainly submitted to cognitive demands at work. The most reported diagnosed diseases were: muscleskeletal diseases (and lesions), neurological (including emotional disturbances), respiratory, digestive, skin and cardiovascular diseases. The analysis of the logistic regression models showed
}

Correspondência para/Correspondence to: Silvia Meirelles Bellusci

Av. Dr. Arnaldo, 715

01246-904 São Paulo, SP - Brasil

E-mail: silviabellusci@uol.com.br
*Baseado na dissertação de mestrado apresentada à Faculdade de Saúde Pública da USP, 1998.

**Aluna de pós-graduação da Faculdade de Saúde Pública Edição subvencionada pela Fapesp (Processo ${ }^{\circ} .98 / 13915-5$ ).

Recebido em 27.11.1998. Reapresentado em 7.4.1999. Aprovado em 16.6.1999. (Processo 520605/96-9). 
that: female workers, those with longer time on the job and job title of operational helper, increase the odds ratio to present low or moderate WAI.

Discussion

The results point out the need to improve the working conditions. It is suggested the implementation of Specialized Safety Engineering and Occupational Medicine Service, as it is demanded by the Federal Law 6,514 of 1977.

\section{INTRODUÇÃO}

A evolução demográfica mostra o envelhecimento da população em geral, justificando a preocupação com o envelhecimento funcional (entendido como perda de capacidade para o trabalho) da classe trabalhadora como segmento dessa população. Levy e Pagliaro ${ }^{10}$, afirmam que “... de 1980 a 1991, enquanto a razão de crescimento da população total foi $1,94 \%$, a do grupo em idade de trabalho foi $2,56 \%$ e, a dos idosos, $2,91 \%$. O grupo mais jovem teve aumento de apenas $0,79 \%$, perdendo população em termos absolutos na década de 90 . O efeito demográfico desta situação é o envelhecimento da população em idade de trabalho e conseqüentemente da força de trabalho...".

A Organização Mundial da Saúde (OMS) ${ }^{11}$ tem demonstrado preocupação com a questão do envelhecimento relacionado ao trabalho e reconhece que modificações nos vários sistemas do corpo humano levam a uma diminuição gradativa na eficácia de cada um deles, com diminuição na capacidade funcional dos indivíduos que pode gerar conflitos entre a capacidade funcional e as exigências do trabalho. Segundo Ilmarinen et al. ${ }^{4}$ e Ilmarinen ${ }^{5,6,7}$, quando o trabalhador tem mais experiência nas tarefas que executa, as exigências do trabalho, especialmente as mentais, tendem a aumentar, podendo levar ao envelhecimento funcional precoce.

As exigências e os fatores de estresse no trabalho precisam estar equilibrados com a capacidade dos trabalhadores para que eles não envelheçam funcionalmente. Há necessidade de uma avaliação contínua dos agentes que desencadeiam sintomas, lesões e doenças e das melhorias das condições de trabalho, procurando soluções para incrementar o equilíbrio da relação entre capacidade e demandas do trabalho. Essas soluções são baseadas em estudos sobre o ambiente de trabalho, as alterações fisiológicas, as mudanças na capacidade para o trabalho, e, em especial, na influência da organização e dos aspectos físicos e ergonômicos no trabalho. É enfatizada a necessidade de flexibilização das tarefas e a identificação de requisitos específicos para promover a saúde na população de trabalhadores que perderam a capacidade funcional para o trabalho. Também são recomendados que se leve em conta o estilo de vida e as condições de trabalho, com o objetivo de otimizar a capacidade funcional e a saúde de trabalhadores. Ao mesmo tempo promover eficiência econômica e produtiva para garantir que a habilidade e experiência sejam totalmente utilizadas.

Na prática, a capacidade para o trabalho precisa ser avaliada para identificar seu declínio em estágio prematuro, acompanhar os efeitos das medidas de prevenção e reabilitação, e para avaliar a incapacidade para o trabalho (Tuomi et al) ${ }^{14}$.

O objetivo do presente trabalho é avaliar o envelhecimento funcional (capacidade para o trabalho) associado às condições de trabalho.

\section{MÉTODOS}

Foram convidados para participar do estudo todos os servidores ativos, em exercício de suas funções, do Tribunal Regional Federal, da $3^{a}$ Região, São Paulo.

O trabalho ali realizado consiste, basicamente, de tarefas relacionadas ao andamento jurídico de processos movidos entre a União e os cidadãos e as relacionadas à administração dos recursos destinados a esse fim. Desde a instalação do TRF $3^{a}$ Região, em 1989, até final de 1996, foram distribuídos 636.882 processos, dos quais 239.125 estavam em andamento ao final de 1996, nos 27 gabinetes de Juiz ${ }^{12}$.

\section{Instrumento de Coleta e Análise dos Dados}

Para avaliar a capacidade para o trabalho utilizou-se o questionário auto-aplicável, denominado Índice de Capacidade para o Trabalho (ICT) (Tuomi et al) $)^{14}$, para ser utilizado em serviços de saúde do trabalhador. Entende-se por capacidade para o trabalho quão bem um trabalhador é capaz de realizá-lo.

As ocupações foram analisadas em relação às cargas de trabalho através do método de Rohmert e Landau ${ }^{13}$, para avaliação ergonômica do trabalho, classificandoas de acordo com as exigências físicas, mentais, ambientais, das tarefas e organizacionais.

Entre dezembro de 1996 e março de 1997, os questionários foram distribuídos pela pesquisadora e preenchidos pelos próprios servidores. A avaliação ergonômica do trabalho foi realizada no primeiro trimestre de 1997 através de coleta de dados por entrevistas individuais ou em grupo e observação do local de trabalho. 
Uma fase piloto foi realizada e 100 questionários foram distribuídos para mulheres com mais de 35 anos, com escolaridade mínima de quarta série do primeiro grau e escolhidas por sorteio. Responderam ao questionário 73 servidoras, que não participaram da segunda fase da coleta de dados.

\section{PopulaçãoEstudada}

No mês de janeiro de 1997, existiam no Tribunal Regional Federal (São Paulo) 1.144 servidores ativos, aos quais foram distribuídos questionários autoaplicáveis. Deste total, foram obtidos 807 respostas (70,5\%); dos restantes 337 (29,5\%), 73 já haviam participado do pré-teste e 264 estavam exercendo outras funções (36) ou se encontravam ausentes (228) por vários motivos: licenças, férias ou licenciamento para prestação de serviço em outro órgão.

\section{Análise Estatística e Variáveis Estudadas}

Caracterizou-se as variáveis como: variável dependente (y) = índice de capacidade para o trabalho - ICT; variáveis independentes $(\mathrm{x})=$ idade, sexo, estado conjugal, escolaridade, tempo de serviço no tribunal, cargo que ocupa, função e local de trabalho (lotação).

Os dados foram analisados através de: teste de associação pelo $X^{2}$ ou Fisher para avaliar o relacionamento entre Índice de Capacidade para o Trabalho e as características demográficas, regressão logística para se obter informação sobre a correlação entre o Índice de Capacidade para o Trabalho e as variáveis independentes.

A variável ICT foi transformada em variável "dummy" para a análise de regressão logística. Foi dado ponto de corte para o ICT entre o ICT moderado e bom (escore $>36,5$ ) e ICT baixo ou moderado (escore $=<36,5$ ), sendo a probabilidade de $\mathrm{Y}=1$ a probabilidade de um funcionário apresentar ICT baixo ou moderado ( $<36,5$ pontos). Para elaborar o modelo preditivo do ICT, através da análise de regressão logística, fez-se inicialmente a análise univariada para a seleção das variáveis. Foram utilizados os programas: Epi Info 6.4, Multlr - Multiple Logistic Regression by Unconditional and Conditional Methods, Excel 5.0, Windows e Word 6.0.

O desenho de investigação que se aplicou a este trabalho é o transversal.

\section{RESULTADOS}

\section{Características da População Estudada}

A idade da população estudada variou de 21 a 70 anos, sendo a média 36 anos e 2 meses (desvio padrão $8,7)$. A idade mediana foi 34 anos e $75 \%$ dos servidores tinham até 41 anos.
A escolaridade mediana dos servidores é superior completa (mínimo de 14 a 15 anos de formação) e representa $75 \%$ dos servidores.

O tempo médio de serviço nesse local foi de 3 anos e 10 meses. Apenas $3(0,4 \%)$ dos servidores entre os 807 pesquisados tinham menos de 1 ano de trabalho no TRF e $34(4,2 \%)$ já haviam completado 8 anos de trabalho, sendo que a moda era 7 anos $(210(26,0 \%)$ servidores) e a mediana 3 anos.

Em relação à lotação do servidor no TRF, 366 $(45,4 \%)$ eram lotados em Gabinetes de Juiz, 28 (3,5\%) na Diretoria Geral, $135(16,7 \%)$ na Secretaria Judiciária, $103(12,8 \%)$ na Secretaria de Administração, 68 (8,4\%) na Secretaria de Recursos Humanos, 21 (2,6\%) na Secretaria de Planejamento, Orçamento e Finanças e 86 (10,7\%) na Secretaria de Documentação e Informática. Os servidores estudados ocupavam os seguintes cargos: técnico judiciário $(\mathrm{n}=138-17,1 \%)$, auxiliar judiciário $(\mathrm{n}=302$ - 37,4\%), atendente judiciário $(\mathrm{n}=123-15,2 \%)$, agente de segurança judiciário $(n=71-8,8 \%)$, auxiliar operacional de serviços diversos $(n=63-7,8 \%)$, digitador e taquígrafo $(\mathrm{n}=25-3,1 \%)$, outros cargos $(\mathrm{n}=85-10,5 \%)$. Exercendo funções de chefia, havia $127(15,7 \%)$ servidores.

Em relação ao predomínio de exigências no trabalho, $23(2,9 \%)$ dos servidores estavam expostos a predomínio de exigências físicas, $595(73,7 \%)$ a exigências cognitivas e $189(23,4 \%)$ a exigências mistas (físicas e cognitivas).

\section{Índice de Capacidade para o Trabalho}

A Tabela 1 apresenta a distribuição do ICT, segundo sexo, idade, estado conjugal, escolaridade, tempo de serviço, cargo, lotação, predominância de exigência no trabalho e função, na população estudada.

As variáveis sexo, idade, estado conjugal, tempo de serviço, cargo de atendente judiciário e cargo de auxiliar operacional de serviços diversos apresentaram associação significativa com perda de capacidade para o trabalho.

\section{Perfil de Doenças}

A Figura 1 mostra a distribuição das lesões e doenças apresentadas com maior freqüência, através de respostas à pergunta sobre a opinião do servidor (OS) sobre quais das lesões por acidentes ou doenças citadas no questionário ele apontava como ocorrência naquele momento. Além disso, o servidor deveria responder também se essas ocorrências haviam sido diagnosticadas pelo médico (DM). 
Tabela 1 - Distribuição do Índice de Capacidade para o Trabalho, segundo características de servidores do Tribunal Regional Federal, 3ª̂̉ Região, 1997.

\begin{tabular}{|c|c|c|c|c|c|c|c|c|c|c|c|c|}
\hline \multirow{3}{*}{ Variável } & \multirow{3}{*}{ Categoria } & \multicolumn{6}{|c|}{ Índice } & & & & & \multirow{3}{*}{$\begin{array}{l}\text { valor } \\
\text { de p* }\end{array}$} \\
\hline & & \multicolumn{2}{|c|}{ ótimo } & \multicolumn{2}{|c|}{ bom } & \multicolumn{2}{|c|}{ moderado } & \multicolumn{2}{|c|}{ fraco } & \multicolumn{2}{|c|}{ total } & \\
\hline & & $\mathrm{n}^{\mathrm{o}}$ & $\%$ & $\mathrm{n}^{\underline{0}}$ & $\%$ & $n^{\circ}$ & $\%$ & $\mathrm{n}^{\mathrm{o}}$ & $\%$ & $n^{0}$ & $\%$ & \\
\hline \multirow[t]{2}{*}{$\overline{\operatorname{sexo}}$} & masculino & 181 & 48,3 & 147 & 39,2 & 44 & 11,7 & 3 & 0,8 & 375 & 46,5 & 0,0002 \\
\hline & feminino & 133 & 30,8 & 204 & 47,2 & 92 & 21,3 & 3 & 0,7 & 432 & 53,5 & \\
\hline \multirow[t]{3}{*}{ idade } & $21 \mid-40$ anos & 237 & 40,1 & 260 & 44,0 & 90 & 15,2 & 4 & 0,7 & 591 & 73,2 & 0,0690 \\
\hline & $40-50$ anos & 53 & 38,1 & 52 & 37,4 & 32 & 23,0 & 2 & 1,4 & 139 & 17,2 & \\
\hline & 50 anos e mais & 28 & 36,4 & 35 & 45,5 & 14 & 18,2 & 0 & 0,0 & 77 & 9,5 & \\
\hline \multirow{3}{*}{$\begin{array}{l}\text { estado } \\
\text { conjugal }\end{array}$} & solteiro & 116 & 39,3 & 134 & 45,4 & 44 & 14,9 & 1 & 0,3 & 295 & 36,6 & 0,0113 \\
\hline & casado, vive comp. & 184 & 42,2 & 177 & 40,6 & 72 & 16,5 & 3 & 0,7 & 436 & 54,0 & \\
\hline & separ., divorc., viúvo & 14 & 18,4 & 40 & 52,6 & 20 & 26,3 & 2 & 2,6 & 76 & 9,4 & \\
\hline \multirow{2}{*}{$\begin{array}{l}\text { escola- } \\
\text { ridade }\end{array}$} & $>2^{\circ}$ Grau comp. & 303 & 39,4 & 330 & 42,9 & 130 & 16,9 & 6 & 0,8 & 769 & 95,3 & 0,7926 \\
\hline & $=<2^{\circ}$ Grau comp. & 11 & 28,9 & 21 & 55,3 & 6 & 15,8 & 0 & 0,0 & 38 & 4,7 & \\
\hline \multirow{4}{*}{$\begin{array}{l}\text { tempo } \\
\text { de } \\
\text { serviço }\end{array}$} & $0 \mid-2$ anos & 110 & 46,0 & 105 & 43,9 & 23 & 9,6 & 1 & 0,4 & 239 & 29,6 & 0,0006 \\
\hline & $2 \mid-4$ anos & 101 & 37,1 & 120 & 44,1 & 49 & 18,0 & 2 & 0,7 & 272 & 33,7 & \\
\hline & $4 \mid-6$ anos & 17 & 39,5 & 15 & 34,9 & 11 & 25,6 & 0 & 0,0 & 43 & 5,3 & \\
\hline & 6 anos e mais & 86 & 34,0 & 111 & 43,9 & 53 & 20,9 & 3 & 1,2 & 253 & 31,4 & \\
\hline \multirow[t]{4}{*}{ cargo } & outros cargos** & 258 & 37,7 & 298 & 43,6 & 122 & 17,8 & 6 & 0,9 & 684 & 84,8 & 0,0104 \\
\hline & atendente judiciário & 56 & 45,5 & 53 & 43,1 & 14 & 11,4 & 0 & 0,0 & 123 & 15,2 & \\
\hline & outros cargos*** & 300 & 40,3 & 321 & 43,1 & 118 & 15,9 & 5 & 0,7 & 744 & 7,8 & 0,0090 \\
\hline & AOSD*** & 14 & 22,2 & 30 & 47,6 & 18 & 28,6 & 1 & 1,6 & 63 & 92,2 & \\
\hline \multirow[t]{2}{*}{ lotação } & outras lotações***** & 259 & 38,5 & 288 & 42,9 & 119 & 17,7 & 6 & 0,9 & 672 & 83,3 & 0,0984 \\
\hline & secret. judiciária & 55 & 40,7 & 63 & 46,7 & 17 & 12,6 & 0 & 0,0 & 135 & 16,7 & \\
\hline exigência & pred. exig. física & 7 & 30,4 & 14 & 60,9 & 2 & 8,7 & 0 & 0,0 & 23 & 2,9 & 0,4500 \\
\hline & pred. exig. cognit & 232 & 39,0 & 260 & 43,7 & 98 & 16,5 & 5 & 0,8 & 595 & 73,7 & \\
\hline trabalho & exigência mista & 75 & 39,7 & 77 & 40,7 & 36 & 19,0 & 1 & 0,5 & 189 & 23,4 & \\
\hline \multirow[t]{2}{*}{ função } & chefia $\approx$ & 59 & 46,5 & 48 & 37,8 & 20 & 15,7 & 0 & 0,0 & 127 & 15,7 & 0,6002 \\
\hline & $\begin{array}{l}\text { sem função de chefia } \\
\text { e sem função }\end{array}$ & 255 & 37,5 & 303 & 44,6 & 116 & 17,1 & 6 & 0,9 & 680 & 84,3 & \\
\hline \multicolumn{2}{|l|}{ Total } & 314 & 38,9 & 351 & 43,5 & 136 & 16,9 & 6 & 0,7 & 807 & 100,0 & \\
\hline
\end{tabular}

* nível descritivo pelo teste de associação do $x^{2}$ de Fisher ** todos servidores, exceto atendente judiciário

*** todos servidores, exceto AOSD

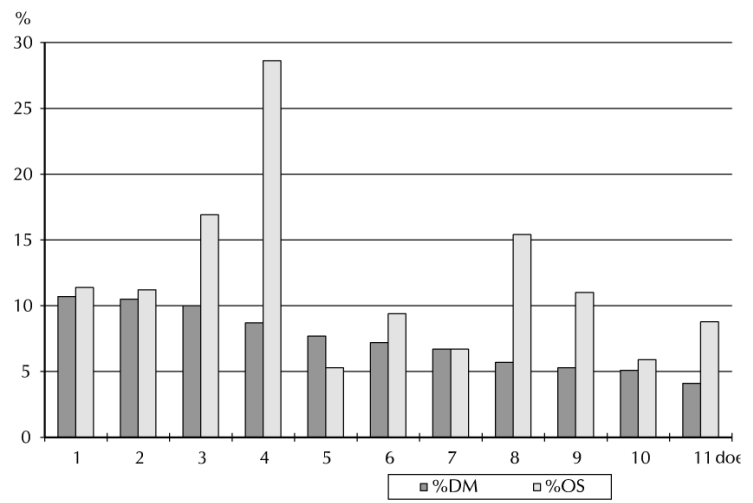

Figura 1 - Percentagem de lesões ou doenças mais freqüentes, com diagnóstico médico ou na opinião do servidor, Tribunal Regional Federal, 3aㅗ Região, 1997.

DM-Diagnóstico Médico

1 - alergia, eczema

2 - gastrite ou irritação duodenal

3 - lesão nas costas

4 - distúrbio emocional leve

5 - hipertensão arterial

6 - doença da parte inferior das

costas com dores freqüentes
OS - Opinião do Servidor

7 - lesão nos braços e mãos

8 - infecções repetidas do trato respiratório

9 - doença parte superior das costas e pescoço

10 - obesidade

11 - lesão nas pernas ou pés
**** AOSD - auxiliar operacional de serviços diversos ***** todos servidores, exceto os lotados na secretaria judiciária

As alergias dermatológicas foram citadas por $86(10,7 \%)$ servidores e rinite alérgica por 46 (5,7\%). Distúrbio emocional leve com diagnóstico médico, foi referido por $70(8,7 \%)$ servidores, enquanto $231(28,6 \%)$ referem sentir seus sintomas. Lesão nas costas, distúrbio emocional leve, lesão nos braços e mãos, doença da parte superior das costas ou região do pescoço, obesidade e doença da parte inferior das costas com dores freqüentes são citados com mais frequiência quando a pergunta se refere a possuir a doença na opinião do próprio servidor. Tal situação se inverte quando o questionamento é sobre a hipertensão arterial: há mais indivíduos com diagnóstico médico dessa doença e menos indivíduos que admitem portá-la. Tumores, doenças hematológicas, defeitos congênitos e outros problemas ou doenças foram os itens menos citados, tanto no quesito diagnóstico médico quanto na opinião do servidor.

\section{Análise Ergonômica do Trabalho}

As figuras 2, 3 e 4 mostram resumos explicativos dos dados levantados pela análise ergonômica do trabalho dos cargos de técnico judiciário, auxiliar judiciário e atendente judiciário, respectivamente. 


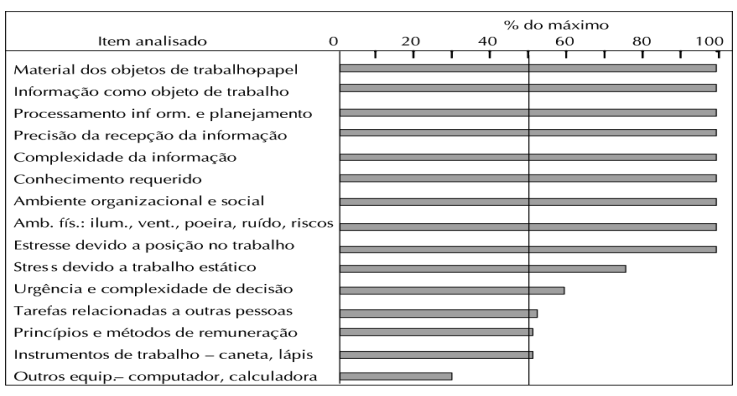

Figura 2 - Descrição dos itens mais significativos ou freqüentes na análise ergonômica do cargo de técnico judiciário.

O técnico judiciário faz uso intensivo da informação, principalmente no que diz respeito à sua complexidade, à pressão de tempo na tomada de decisões e à educação e conhecimentos específicos exigidos para o desempenho de suas tarefas. Da mesma forma é significativo o planejamento e a organização do uso, codificação, classificação, assim como associação e análise da informação, que exigem precisão em sua recepção. Isto revela o predomínio de exigências cognitivas para a realização do trabalho.

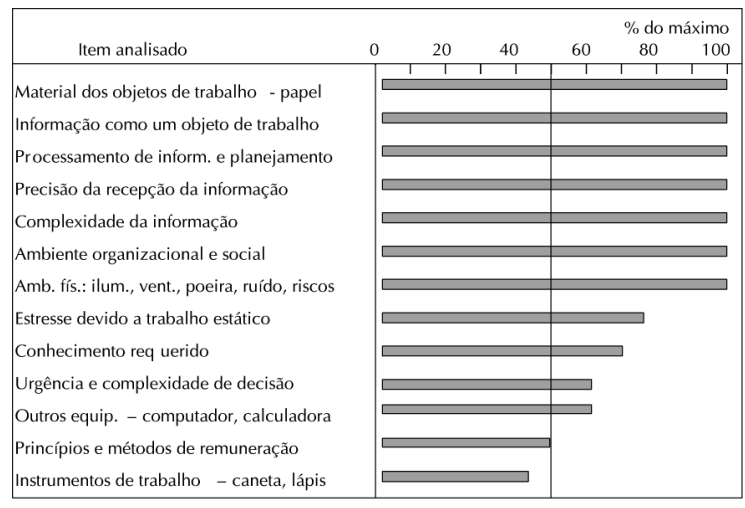

Figura 3 - Descrição dos itens mais significativos ou freqüentes na análise ergonômica do cargo de auxiliar judiciário.

O auxiliar judiciário faz uso da informação, porém suas atividades estão associadas à operação de coleta e preparo dos dados. O planejamento e a organização das tarefas, a codificação e a classificação da informação, a necessidade de associação e análise, que exigem precisão na recepção da informação, também estão presentes na maior parte do seu período de trabalho, caracterizando predomínio de exigências cognitivas no trabalho.

$\mathrm{O}$ atendente judiciário é, entre os três cargos analisados, aquele que faz uso menos intenso da informação. É o que está mais exposto às exigências físicas, principalmente no que diz respeito a movimentos ativos, já que nesse cargo o servidor realiza

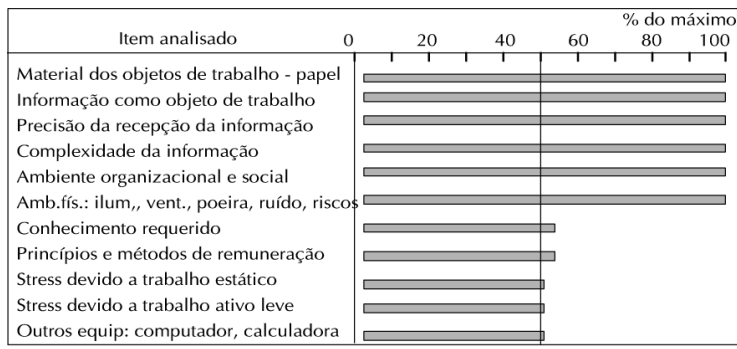

Figura 4 - Descrição dos itens mais significativos ou freqüentes na análise ergonômica do cargo de atendente judiciário.

tarefas relacionadas ao movimento dos processos dentro e fora dos gabinetes. Isto caracteriza predomínio de exigências mistas, ou seja, este cargo se submete a exigências físicas e cognitivas.

O auxiliar operacional de serviços diversos foi admitido para executar limpeza, sob coordenação e orientação, tarefas operacionais complementares nas áreas de escritório, copa e cozinha e conservação. Por ter havido terceirização desse tipo de tarefas operacionais, essa categoria passou a realizar atividades similares às do atendente judiciário. Entretanto, informalmente, a situação do auxiliar operacional de serviços diversos no trabalho é pior que a do atendente judiciário. Estes últimos se submetem a realizar tarefas mais árduas, do ponto de vista físico (tais como carregar processos, operar máquinas fotocopiadoras) que o atendente judiciário. Além disso, também se submetem a exigências cognitivas.

Os três cargos exigem o uso de equipamentos para a transformação da informação, tais como: lápis, caneta, máquina de escrever e computador. No entanto, o auxiliar judiciário e o atendente judiciário são os que fazem mais uso do computador; o técnico judiciário realiza por mais tempo trabalho estático, sentado, curvado sobre livros, processos ou escritos.

As tarefas dos técnicos, auxiliares e atendentes são desenvolvidas em sala contígua à do Juiz, medindo aproximadamente $100 \mathrm{~m}^{2}$, de uso comum a um número de 15 a 17 funcionários, contendo 15 a 17 mesas de escritório e mesas para computador.

As paredes são forradas de prateleiras metálicas, abertas, que comportam grandes quantidades de processos (8.800 em média), cujas capas são de papel tipo cartolina, frágeis para o fim a que se destinam (manipulação, guarda e transporte de documentos) e geralmente se apresentam deterioradas. Suas cores indicam o conteúdo básico da petição. Dessa forma, ao se adentrar em uma secretaria de Juiz, depara-se com paredes lotadas de processos geralmente de uma 
mesma cor, mesas justapostas com muitos processos sobre elas e mesmo no chão ao seu redor. A falta de espaço para circulação das pessoas é nítida. As janelas freqüentemente permanecem fechadas para evitar a entrada de ruído externo (o prédio se localiza na zona central de São Paulo) ou excesso de movimentação do ar, que move papéis em uso sobre as mesas. A ventilação natural deficiente é agravada por constantes reformas, necessárias para garantir a segurança do prédio, o que faz com que as janelas não possam ser abertas para não entrar poeira proveniente das obras de restauração. Por se tratar de prédio tombado pelo patrimônio histórico (CONDEPHAAT) e construído na década de 30, suas instalações elétricas não permitem o uso de condicionadores de ar.

Em relação ao mobiliário, observou-se que as mesas utilizadas não oferecem possibilidade de regulagem de altura do seu tampo, sendo portanto inadequadas para uso às pessoas de porte mais alto $(>1,75 \mathrm{~m}) \mathrm{ou}$ mais baixo ( $<1,55 \mathrm{~m})$. A grande maioria das mesas apresenta bordas em ângulo de $90^{\circ}$, provocando desconforto nas articulações do cotovelo e na pele do antebraço. O mesmo ocorre com as mesas utilizadas para apoio dos computadores e seus teclados, que, sem regulagem de altura do tampo do monitor e do teclado, e pequenas para o fim a que se destinam, impedem o apoio de braços e punhos. Também levam os usuários a manter a cabeça para o alto ou para baixo, já que o monitor, salvo exceções, se encontra mais alto ou mais baixo do que a altura adequada para seu usuário. A maioria das cadeiras tem ajuste para altura do assento e do encosto. Porém, esses ajustes são de difícil acesso e deixam de ser regulados pelo usuário. Devido à falta de espaço, muitas vezes reguladores de voltagem ou caixas de papel para impressão são colocados sob os tampos das mesas, impedindo a movimentação livre das pernas e pés. Os servidores permanecem a maior parte do período de trabalho sentados, com o corpo curvado para frente, os braços sobre a mesa, manipulando processos, livros, jornais, lendo, escrevendo, analisando, decidindo ou digitando.
Esse é o quadro: o indivíduo sentado, com os pés sem apoio ou as pernas sem movimentação livre, as costas e o pescoço arqueados para frente, a cabeça arqueada para cima ou para frente (dependendo da altura da mesa ou do monitor), os braços e os punhos sem possibilidade de apoio sobre a mesa, sendo mantidos em pleno ar às custas da musculatura posterior, ou apoiados sobre desagradável borda da mesa em ângulo de $90^{\circ}$. Além desses fatores, os indivíduos estão envolvidos em tarefas de alta responsabilidade, para ser executada em curto espaço de tempo.

\section{Análise univariada de regressão logística}

As variáveis selecionadas para a elaboração do modelo multivariado foram aquelas que, na análise univariada, apresentaram $\mathrm{p}<0,15$ : sexo $(\mathrm{p}=0,0001)$, tempo de serviço $(\mathrm{p}=0,0020)$, cargo $(\mathrm{p}=0,0107)$, estado conjugal $(\mathrm{p}=0,0156)$, idade $(\mathrm{p}=0,0690)$ e lotação $(\mathrm{p}=0,1425)$. Ficam excluídas as variáveis função $(\mathrm{p}=0,5466)$ e escolaridade $(\mathrm{p}=0,7614)$.

\section{Análise multivariada de regressão logística}

O processo de modelagem foi iniciado pela variável sexo, seguida pela introdução da variável tempo de serviço e cargo. Essas permaneceram no modelo, pois mantiveram a significância estatística do modelo e as suas próprias significâncias. A inclusão da variável estado conjugal provocou perda da significância estatística da variável cargo e de sua própria significância, sendo excluída do modelo. As variáveis idade e lotação provocaram perda de significância para a variável cargo e as suas próprias significâncias, e, conseqüentemente, não foram mantidas no modelo.

Em relação à variável idade, optou-se por realizar a análise de duas formas: com sua exclusão, já que não se mostrou significativa, e com a sua inclusão, considerando sua importância epidemiológica (Tabela 2).

$$
\text { Sendo } \mathrm{p}=\frac{1}{1+\mathrm{e}^{-\mathrm{f}(\mathrm{xi})}}=\mathrm{a} \text { probabilidade de } \mathrm{Y}=1 \text {, ou }
$$

Tabela 2 - Análise multivariada final dos fatores de risco do Índice de Capacidade para o Trabalho, através do modelo de análise de regressão logística, em servidores do Tribunal Regional Federal, 3ª Região, 1997.

\begin{tabular}{|c|c|c|c|c|c|c|}
\hline Variáveis & Categoria & Coef. $\left(b_{i}\right)$ & $\mathrm{OR}_{\mathrm{aj}}$ & $\mathrm{IC}_{95 \%}(\mathrm{OR})$ & p(categoria) & p(variável) \\
\hline sexo & $\begin{array}{l}\text { masculino } \\
\text { feminino }\end{array}$ & $\begin{array}{c}\text { referência } \\
0,7509\end{array}$ & 2,119 & {$[1,432 ; 3,135]$} & 0,0002 & 0,0000 \\
\hline $\begin{array}{l}\text { tempo de } \\
\text { serviço }\end{array}$ & $\begin{array}{lc}0 & 2 \text { anos } \\
2 & 4 \text { anos } \\
4 & 6 \text { anos } \\
6 & \text { anos e mais }\end{array}$ & $\begin{array}{c}\text { referência } \\
0,6301 \\
1,0195 \\
0,8895\end{array}$ & $\begin{array}{l}1,878 \\
2,772 \\
2,434\end{array}$ & $\begin{array}{l}{[1,116 ; 3,159]} \\
{[1,232 ; 6,234]} \\
{[1.448 ; 4,090]}\end{array}$ & $\begin{array}{l}0,0176 \\
0,0137 \\
0,0008\end{array}$ & 0,0000 \\
\hline cargo & $\begin{array}{l}\text { outros cargos* } \\
\text { AOSD }\end{array}$ & $\begin{array}{c}\text { referência } \\
0,5982\end{array}$ & 1,819 & {$[1,008 ; 3,282]$} & 0,0470 & 0,0542 \\
\hline
\end{tabular}

$\mathrm{OR}_{\mathrm{a} ;}$ : odds ratio ajustada

Significância do modelo final $p=0,0542$

* todos servidores, exceto AOSD 
seja, a probabilidade de um servidor ter ICT moderado ou baixo, onde $\mathrm{f}\left(\mathbf{x}_{\mathrm{i}}\right)=$ onde $\mathrm{f}\left(\mathbf{x}_{\mathrm{i}}\right)=-2,6388+0,7509 \mathrm{SEXOi}$ $+0,6301 \mathrm{TSERV} 42 \mathrm{i}+1,0195 \mathrm{TSERV} 43 \mathrm{i}+$ 0,8895TSERV44i +0,5982 CARGO85i.

Os indivíduos com maior probabilidade de apresentar ICT moderado ou baixo são mulheres que trabalharam no TRF de $4 \mid-6$ anos, no cargo de auxiliar operacional de serviços diversos $\left({ }^{\wedge} \mathrm{p}=0,43\right)$. São seguidas por aquelas com 6 anos ou mais de trabalho $(\wedge \mathrm{p}=0,40)$.

A introdução da variável idade provoca perda de significância para a categoria da variável cargo ( $\mathrm{p}=0,0995)$, alterando o intervalo de confiança de sua "odds ratio" $[0,908 ; 3,060]$. Ao mesmo tempo, em relação à variável idade, apenas a categoria de $40 \mid-50$ anos é significativa $(\mathrm{p}=0,0317)$.

A probabilidade de um servidor ter ICT moderado ou baixo, se for considerada a introdução da variável idade no modelo, é: $\mathrm{f}\left(\mathbf{x}_{\mathrm{i}}\right)=-2,7499+0,7734$ SEXOi + 0,6466TSERV42i +0,9868 TSERV43i +0,8681TSERV44i + 0,5108 CARGO85i +0,5111 IDADE2i+0,1120 IDADE3i.

\section{DISCUSSÃO}

A variável sexo apresentou associação significativa com a perda da capacidade de trabalho. Esse resultado é similar ao publicado por outros autores que

estudaram as relações entre condições de trabalho e saúde, comparando os gêneros masculino e feminino. Com relação ao maior risco apresentado pelas mulheres, tal achado tem múltiplas causas, sendo freqüentemente citado (Kandolin ${ }^{8}$, Hall $^{3}$ ).

Alergias dermatológicas e rinite alérgica, relatadas pelos servidores, indicam exposição no trabalho a alérgenos (ácaros, fungos e poeiras) provenientes do contato contínuo com grandes quantidades de papéis.

Com relação à hipertensão arterial, alguns autores encontraram associações significativas entre a ocupação e esta doença. Cordeiro et al. $^{2}$, ao estudarem uma população de motoristas urbanos e cobradores, encontraram prevalência de $30 \%$ para hipertensão. Klein e Araújo ${ }^{9}$, encontraram prevalências entre 2,9\% e $16 \%$, dependendo do grupo de trabalhadores estudados. Na presente população, a porcentagem relatada de diagnósticos de hipertensão é de 7,7\%.

Os resultados do ICT mostram que $493(61,1 \%)$ dos 807 servidores que participaram da pesquisa necessitam ter sua capacidade para o trabalho melhorada ou restaurada. Entre eles, as doenças mais freqüentes são: alergia, gastrite ou irritação duodenal, lesão nas costas, distúrbio emocional leve, hipertensão arterial, lesão nos braços e mãos, infecções repetidas do trato respiratório, doença da parte superior das costas ou região do pescoço, obesidade, lesão nas pernas ou pés e doença da parte inferior das costas com dores freqüentes.

A análise de regressão logística mostra associação do ICT com:

a) o sexo: as mulheres têm 2,2 vezes mais chance de ter o ICT baixo ou moderado do que os homens;

b) tempo de serviço: quanto maior o tempo de serviço, maior o risco; trabalhar de $2 \mid \_4$ anos no TRF $3^{\text {a }}$ Região aumenta em 1,9 vezes a chance de ter ICT baixo ou moderado, enquanto trabalhar de $4 \mid-6$ anos aumenta 2,8 vezes e, com mais de 6 anos, o risco é de 2,4 vezes;

c) auxiliar operacional de serviços diversos: ocupar esse cargo aumenta em 1,8 vezes a chance de o indivíduo ter ICT baixo ou moderado; esse resultado converge com o encontrado na análise ergonômica do trabalho, que evidenciou que a ocupação de auxiliar operacional de serviços diversos está exposta a maior número de estressores ocupacionais e por maior tempo,

d) idade: a faixa etária compreendida entre $40-50$ anos apresenta maior probabilidade de ter o ICT moderado ou baixo; essa situação denota a possibilidade de, em faixas mais altas, os indivíduos com perda de capacidade para o trabalho já terem se afastado voluntária ou involuntariamente do serviço, o que não foi detectado por ser este um estudo transversal e poder apresentar o chamado efeito do trabalhador sadio.

A análise ergonômica do trabalho, o ICT, o perfil de doenças e as análises univariadas e multivariadas de regressão logística indicam que as doenças mais comumente apresentadas pelo servidores podem guardar nexo causal com as condições inadequadas nas quais o trabalho é realizado.

Uma situação peculiar do estudo transversal em epidemiologia ocupacional é a que exclui o possível doente (27 estavam afastados por motivo de doença), levando ao efeito do trabalhador sadio (Checkoway et al. ${ }^{1}$ ). Essa é uma situação que pode subestimar o tamanho dos riscos oferecidos pelo processo de trabalho, porque os mais afetados não conseguem manter-se no emprego, afastando-se por licenças para tratamento de saúde, por demissões e por outros motivos. Por ser este um estudo transversal, não se pode prever como os aspectos de saúde evoluirão, nem 
como eram anteriormente. $\mathrm{O}$ ideal seria repetir a avaliação da capacidade para o trabalho periodicamente e analisar as possíveis mudanças para uma efetiva intervenção na prevenção dos danos à saúde.

No presente trabalho não foi possível investigar variáveis relacionadas ao estilo de vida, como hábito de fumar, ingerir bebida alcoólica, praticar exercício físico e outras. Isso traz uma limitação ao estudo, pois algumas dessas variáveis poderiam ter influência na perda da capacidade para o trabalho.

No entanto, considerando a literatura pertinente, os resultados mostram a necessidade de melhorar as condições de trabalho para garantir a permanência das

\section{REFERÊNCIAS}

1. Checkoway H, Pearce N, Crawford-Brown DJ. Research methods in occupational epidemiology, New York: Oxford University Press; 1989.

2. Cordeiro R, Lima Filho EC, Fischer FC, Moreira Filho DC. Associação da pressão arterial diastólica com o tempo acumulado de trabalho entre motoristas e cobradores. Rev Saúde Pública 1993;27:363-72.

3. Hall EM. Women's work: An Inquiry into the health effects of invisible and visible labor. Stockholm: Akademitryck AB; 1990.

4. Ilmarinen J, Tuomi K, Eskelinen L, Nygard C-H, Huuhtanen P, Klockars M. Background and objectives of the Finnish research project on aging workers in municipal occupations. Scand J Work Environ Health 1991a;17 Suppl. 1:7-11.

5. Ilmarinen J. Aging and work. In: International Scientific Symposium on Aging and Work. Helsinki: Institute of Occupational Health; 1993.

6. Ilmarinen J. Promoting the health and well-being of the older worker: the Finnish experience. In: Investing in older people at work, health education authority. London: Hamilton House; 1994.

7. Ilmarinen J. Aging and work: the role of ergonomics for maintaining work ability during aging. In: Bittner AC, Champney PC. Advances in industrial ergonomics and safety. London: Taylor \& Francis; 1995. pessoas evitando afastamentos temporários ou definitivos precocemente por incapacidade para o trabalho. Para tanto, sugere-se a criação de um Serviço Especializado em Engenharia de Segurança e Medicina do Trabalho, como exige a Lei 6.514 de 1977, que pela Portaria 33, de 27.10.83, do Ministério do Trabalho regulamenta a implantação desse serviço nas empresas.

\section{AGRADECIMENTOS}

Aos servidores forenses, por sua colaboração, e à Professora Dra. Maria do Rosário Dias de Oliveira Latorre, da Faculdade de Saúde Pública da USP, pela orientação nas análises de regressão logística.

8. Kandolin I. Gender, worklife and family responsabilities in Finland and Estonia: Effects on economic and mental and well-being - Research reports 15. People and work. Finnish Inst. Occup. Health; 1997.

9. Klein $\mathrm{CH}$, Araújo JWG. Fumo, bebida alcoólica, migração, instrução, ocupação, agregação familiar e pressão arterial em Volta Redonda, Rio de Janeiro. Cad Saúde Pública 1985;1:160-76.

10. Levy MSF, Pagliaro $H$. The aging of working-age population in Brazil, In: 13th Triennial Congress of the International Ergonomics Association; 1997. Tampere, Finland.

11. Organisation Mondiale de la Santé. Vieillissement et capacité de travail: rapport d'un groupe d'etude de l'OMS. Genève; 1993 (Série de Rapports Techniques, 835).

12. Relatório Geral do Ano Judiciário de 1996. São Paulo: Tribunal Regional Federal da 3ª Região; 1997.

13. Rohmert W, Landau K. A new technique for job analysis. London: Taylor \& Francis; 1983.

14. Tuomi K, Ilmarinen J, Jahkola A, Katajarinne L, Tulkki A. Índice de capacidade para o trabalho. Tradução de Fischer FM et al. Helsinki: Institute of Occupational Health. São Paulo: FSPUSP; 1997. 